\title{
Increasing incidence of diagnosed type 2 diabetes in Taiwan: analysis of data from a national cohort
}

\author{
C.-H. Tseng • C.-P. Tseng • C.-K. Chong • T.-P. Huang • \\ Y.-M. Song $\cdot$ C.-W. Chou $\cdot$ S.-M. Lai $\cdot$ T.-Y. Tai • \\ J.-C. Cheng
}

Received: 21 October 2005 / Accepted: 19 April 2006 / Published online: 21 June 2006

(C) Springer-Verlag 2006

\begin{abstract}
Aims/hypothesis Epidemiological evidence shows an increasing prevalence of type 2 diabetes in Taiwan. The aim of this study was to assess the yearly incidence for this country during 1992-1996.

Subjects and methods Data obtained by telephone interviews of 93,484 diagnosed diabetic patients enrolled in Taiwan's National Health Insurance programme formed the basis of this study. A total of 36,153 incident cases of type 2 diabetes (17,097 men and 19,056 women) were identified and incidence rates calculated. The trends of obesity and parental diabetes were also evaluated.
\end{abstract}

C.-H. Tseng $(\bowtie)$

Department of Internal Medicine,

National Taiwan University Hospital,

7 Chung-Shan South Road,

Taipei, Taiwan, Republic of China

e-mail: ccktsh@ms6.hinet.net

C.-H. Tseng

National Taiwan University College of Medicine,

Taipei, Taiwan, Republic of China

C.-P. Tseng

School of Medical Technology,

Chang Gung University,

Taoyuan, Taiwan, Republic of China

C.-K. Chong

Department of Rehabilitation,

Chang Gung Memorial Hospital,

Taoyuan, Taiwan, Republic of China

\section{T.-P. Huang}

Department of Internal Medicine,

St Martin De Porres Hospital,

Chiayi, Taiwan, Republic of China
Results The overall 5-year incidences for men and women were 187.1 and 218.4 per 100,000 population, respectively. The trends from 1992-1996 were increased for all age groups in men and for most age groups in women. A 2.8-fold increase in incidence was observed for the youngest age group ( $<35$ years), in which the increase in incidence was higher than in the older age groups. Men showed a higher fold increase in incidence than did women (3.5 vs 2.1). Obesity at interview increased from $39.2 \%$ in 1992 to $47.6 \%$ in $1996(p<0.001)$ and was significant for all ages. Parental diabetes showed no yearly change when all patients were analysed together, but there

\section{Y.-M. Song}

Section of Biochemistry, Medical Laboratories,

Taichung Veterans General Hospital,

Taichung, Taiwan, Republic of China

C.-W. Chou

Division of Endocrinology and Metabolism,

Section of Internal Medicine, Chi-Mei Medical Center,

Tainan, Taiwan, Republic of China

S.-M. Lai

Division of Endocrinology and Metabolism,

Department of Internal Medicine,

Shinkong Wu Ho-Su Memorial Hospital,

Taipei, Taiwan, Republic of China

T.-Y. Tai

Division of Gerontology Research

of the National Health Research Institutes,

Taipei, Taiwan, Republic of China

J.-C. Cheng

Department of Medical Laboratory Science and Biotechnology,

China Medical University,

Taichung, Taiwan, Republic of China 
was a trend towards a decrease in the youngest age group $(<35$ years $)$ and a trend towards an increase in the oldest age groups ( $\geq 55$ years).

Conclusions/interpretation An increasing incidence of diagnosed type 2 diabetes was observed for each sex in most age groups in Taiwan, but was most marked in the youngest age group. A parallel increase in obesity was observed with the increasing incidence of diabetes.

Keywords Incidence - National Health Insurance - Obesity · Taiwan · Type 2 diabetes

\section{Abbreviations \\ NHI National Health Insurance}

\section{Introduction}

According to three different surveys in the city of Taipei, the crude prevalence rate of diabetes for those aged $\geq 40$ years was $5.1 \%$ in $1970,7.1 \%$ in 1979 , and $8.2 \%$ in 1986 [1]. However, because of changes in diagnostic criteria, the lack of standardisation in methods and changes in the demographic structure of the population, it was not possible to evaluate whether this trend towards an increase in prevalence truly reflects an increasing incidence of diabetes in Taiwan. Furthermore, the increasing prevalence of diabetes may result from any one or any combination of the following factors: a rise in the incidence rate, a change in the mortality rate in either the diabetic or the non-diabetic population, an earlier age of onset of diabetes, demographic changes, or changes in the ratio of diagnosed to undiagnosed cases [2]. Although there is probably little doubt regarding the increasing prevalence of diabetes [3], whether there is also an increase in incidence is controversial $[2,4,5]$.

The National Health Insurance (NHI) programme is a health care system unique to Taiwan. Joining this health care system is mandatory for every person, as stipulated by law, except for those who are serving in the military or are subject to criminal sanction, etc. [6, 7]. Thus, effective use of its database would allow us to accurately estimate the yearly incidence of diagnosed diabetes in Taiwan. Age, obesity, parental diabetes and race/ethnicity have consistently been shown to be important risk factors for type 2 diabetes $[8,9]$. The purpose of this study was to assess the national incidence trends of diagnosed type 2 diabetes during 1992-1996 (before the revision of the diagnostic criteria for diabetes proposed by the American Diabetes Association in 1997 [10]) in each specific age and sex subgroup in the Taiwanese population and to evaluate the impact of obesity and parental diabetes, by using the data of an epidemiological survey of diagnosed diabetic patients enrolled in the NHI system.

\section{Subjects and methods}

Study subjects

The study was approved by the ethics committee of the Department of Health of Taiwan. Participants were informed of the purpose of the study and consented to be interviewed. Because $>96 \%$ of the total population of Taiwan is covered by the compulsory and country-wide $\mathrm{NHI}$ and the copayment rate is low (5-30\% for inpatient care, and there is a fixed charge of approximately US $\$ 1.40$ 12.4 for outpatient care), almost all diagnosed diabetic patients are enrolled in this system [6,7]. Therefore, the NHI databases of medical benefit claims are suitable for deriving a national sample of diagnosed diabetic patients. The selection of such a national sample has also been described elsewhere [6, 7]. In brief, we identified a total of 256,036 patients from 66 hospitals and clinics located evenly throughout Taiwan, using the codes for the diagnosis of diabetes mellitus defined by the ninth revision of the International Classification of Diseases; i.e. code 250 or the abridged code of A181. The 66 hospitals and clinics were selected so as to give a homogeneous geographical distribution and to include all levels of medical setting. To create a cohort of 90,000 patients (approximately one-sixth of the estimated number of 540,000 diagnosed diabetic patients in Taiwan during the period) for long-term followup, one out of every two identified patients (i.e. 128,572 cases from the 256,036 patients) was randomly selected, assuming a response rate of $70 \%$. Each identified patient was given a unique serial number, and a random sample was created by using the random sample of cases in the statistical package SPSS (Chicago, IL, USA). The total number of diagnosed diabetic patients covered by NHI between July 1997 and June 1998 was 536,159 [11]. This figure was used as the definition of 'all diagnosed diabetic patients' during the study period. The patients in this study were considered to be a subset of the database total of 536,159 because the same codes as those mentioned above were used to identify the patients from the databases of the clinical settings.

\section{Telephone interview}

From 1 March 1995 to 30 April 2002, well-trained interviewers used a structured questionnaire to conduct a telephone survey. There were six interviewers during most time periods and four of them worked from the beginning to the end of the project. Researchers tried to contact subjects up to three times before giving up. The interviewers handed in the interview questionnaires every week, and all returned questionnaires were checked by an assistant and then double-checked by the investigator. 
Table 1 Baseline characteristics of the incident cases of diagnosed type 2 diabetes with National Health Insurance in Taiwan during the period 1992-1996

\begin{tabular}{|c|c|c|c|c|}
\hline Characteristic & Total & Women & Men & $p$ value \\
\hline$n$ & 36,153 & 19,056 & 17,097 & \\
\hline Age at onset (years) & $56.8(11.3)$ & $57.5(10.8)$ & $55.9(11.7)$ & $<0.001$ \\
\hline Duration of diabetes (years) & $4.3(1.8)$ & $4.3(1.8)$ & $4.2(1.8)$ & $<0.05$ \\
\hline BMI at interview $\left(\mathrm{kg} / \mathrm{m}^{2}\right)$ & $24.8(3.6)$ & $24.9(3.7)$ & $24.6(3.4)$ & $<0.001$ \\
\hline Obesity at interview (\%) & 42.8 & 44.0 & 41.5 & $<0.001$ \\
\hline Parent with diabetes $(\%)$ & 25.5 & 23.4 & 27.9 & $<0.001$ \\
\hline
\end{tabular}

Data are means (SD) or percentages

The $p$ values were determined using the $\chi^{2}$ test or Student's $t$-test

Information obtained from the questionnaire

The information obtained from this interview for analysis included age, sex, family history of diabetes, presence or absence of diabetic ketoacidosis at onset of diabetes, requirement and time of insulin use after diabetes diagnosis, the year of diabetes diagnosis, and body height and weight at the time of interview. The BMI at interview was calculated and obesity was defined as BMI $\geq 25 \mathrm{~kg} / \mathrm{m}^{2}$, as suggested for Asian populations [12].

\section{Classification of diabetes}

The classification of type 1 diabetes was based on either of the following two criteria: (1) diabetic ketoacidosis at the onset of diabetes; or (2) requirement for insulin injection within 1 year of diagnosis of diabetes. If a patient was not diagnosed as having type 1 diabetes, he or she was viewed as a patient with type 2 diabetes. Patients diagnosed as having type 1 diabetes were not included in this study. A total of 93,484 (response rate $72.7 \%$ ) patients completed the interview. After excluding 3,528 patients with type 1 diabetes, there were 89,956 patients identified as having type 2 diabetes. Among them, 36,153 patients (19,056 women and 17,097 men) were incident cases diagnosed between the years 1992 and 1996.

Calculation of incidence rates

In Taiwan, a household registration system has been strictly enforced. Therefore, age- and sex-specific population

Table 2 Incidence rates (per 100,000 population) and incident case numbers (in parentheses) by age, sex and calendar year

\begin{tabular}{|c|c|c|c|c|c|c|c|c|}
\hline Age group (years) & Total & 1992 & 1993 & 1994 & 1995 & 1996 & $p^{\mathrm{a}}$ & IRR \\
\hline \multicolumn{9}{|l|}{ Both sexes } \\
\hline$<35$ & $9.3(1,038)$ & $5.3(119)$ & $7.3(164)$ & $8.0(178)$ & $11.3(250)$ & $14.8(327)$ & 0.006 & 2.8 \\
\hline $35-44$ & $144.9(4,221)$ & $98.7(531)$ & $129.6(729)$ & $145.4(853)$ & $167.2(1,012)$ & $176.8(1,096)$ & 0.002 & 1.8 \\
\hline $45-54$ & $547.2(8,923)$ & $402.2(1,230)$ & $552.1(1,723)$ & $570.84(1,843)$ & $610.8(2,047)$ & $586.0(2,080)$ & 0.09 & 1.5 \\
\hline $55-64$ & 937.0 (12 349) & $682.5(1,784)$ & $946.1(2,481)$ & $991.4(2,611)$ & $983.5(2,595)$ & $1,077.8(2,878)$ & 0.053 & 1.6 \\
\hline$\geq 65$ & $725.8(9,622)$ & $492.6(1,182)$ & $671.5(1,696)$ & $739.4(1,968)$ & $799.8(2,219)$ & $882.7(2,557)$ & 0.005 & 1.8 \\
\hline Total & $197.0(36$ 153) & $134.6(4,846)$ & $186.9(6,793)$ & $202.7(7,453)$ & $219.6(8,123)$ & $239.1(8,938)$ & 0.009 & 1.8 \\
\hline \multicolumn{9}{|l|}{ Men } \\
\hline$<35$ & $10.1(582)$ & $5.2(60)$ & $6.7(77)$ & $7.8(90)$ & $13.1(150)$ & $18.0(205)$ & 0.011 & 3.5 \\
\hline $35-44$ & $219.5(2,470)$ & $144.6(287)$ & $185.5(390)$ & $215.0(480)$ & $259.2(617)$ & $272.6(696)$ & 0.001 & 1.9 \\
\hline $45-54$ & $582.6(4,216)$ & $378.7(547)$ & $544.8(790)$ & $586.6(855)$ & $701.4(1,013)$ & $702.2(1,011)$ & 0.013 & 1.9 \\
\hline $55-64$ & $800.7(5,476)$ & $549.2(772)$ & $705.3(1,101)$ & $813.4(1,111)$ & $894.2(1,201)$ & $963.4(1,291)$ & 0.02 & 1.8 \\
\hline$\geq 65$ & $721.4(4,353)$ & 446.3 (492) & $667.9(770)$ & $697.1(843)$ & $818.5(1,028)$ & $928.8(1,220)$ & 0.005 & 2.1 \\
\hline Total & 187.1 (17 097) & $120.2(2,158)$ & $172.7(3,128)$ & $184.6(3,379)$ & $217.9(4,009)$ & $237.6(4,423)$ & 0.004 & 2.0 \\
\hline \multicolumn{9}{|l|}{ Women } \\
\hline$<35$ & $8.4(456)$ & $5.4(59)$ & $8.0(87)$ & $8.1(88)$ & $9.3(100)$ & 11.4 (122) & 0.008 & 2.1 \\
\hline $35-44$ & $161.1(1,751)$ & $127.9(244)$ & $167.5(339)$ & $172.9(373)$ & $171.3(395)$ & $161.5(400)$ & 0.281 & 1.3 \\
\hline $45-54$ & $653.2(4,707)$ & $483.6(683)$ & $649.2(933)$ & $678.6(988)$ & $712.4(1,034)$ & $737.6(1,069)$ & 0.036 & 1.5 \\
\hline $55-64$ & $1,084.1(6,873)$ & $837.5(1,012)$ & $1,114.8(1,380)$ & $1,183.1(1,500)$ & $1,076.2(1,394)$ & $1,193.0(1,587)$ & 0.156 & 1.4 \\
\hline$\geq 65$ & $873.2(5,269)$ & $625.8(690)$ & $803.2(926)$ & $930.2(1,125)$ & $948.3(1,191)$ & $1,017.8(1,337)$ & 0.013 & 1.6 \\
\hline Total & $218.4\left(\begin{array}{l}19 \\
056)\end{array}\right.$ & $157.7(2,688)$ & $212.5(3,665)$ & $233.1(4,074)$ & $233.4(4,114)$ & $252.7(4,515)$ & 0.029 & 1.6 \\
\hline
\end{tabular}

${ }^{\mathrm{a}}$ The trend in $p$ was tested by linear regression

IRR Incidence rate ratio between year 1996 and year 1992 
numbers are very complete and accurate. The data for recent years are available as electronic files [13]. The incidence rates were calculated from the 36,153 patients with type 2 diabetes diagnosed within the 5-year period from 1992 to 1996. The numerators were the incident case numbers within the respective year. The denominators were the background populations divided by 5.74, reflecting the percentage of individuals with health insurance who were interviewed (i.e. 536,159 divided by 93,484). The background populations were the respective mid-year populations of Taiwan [13]. To calculate the average incidence rates for the whole 5-year period, the background populations were the averages of the mid-year populations of the 5 years.

\section{Statistical analyses}

Differences in baseline characteristics between sexes were tested with Student's $t$-test for continuous variables and the $\chi^{2}$ test for categorical variables. The age at onset was divided into the categories $<35,35-44,45-54,55-64$ and $\geq 65$ years. Incidence rates were calculated for each
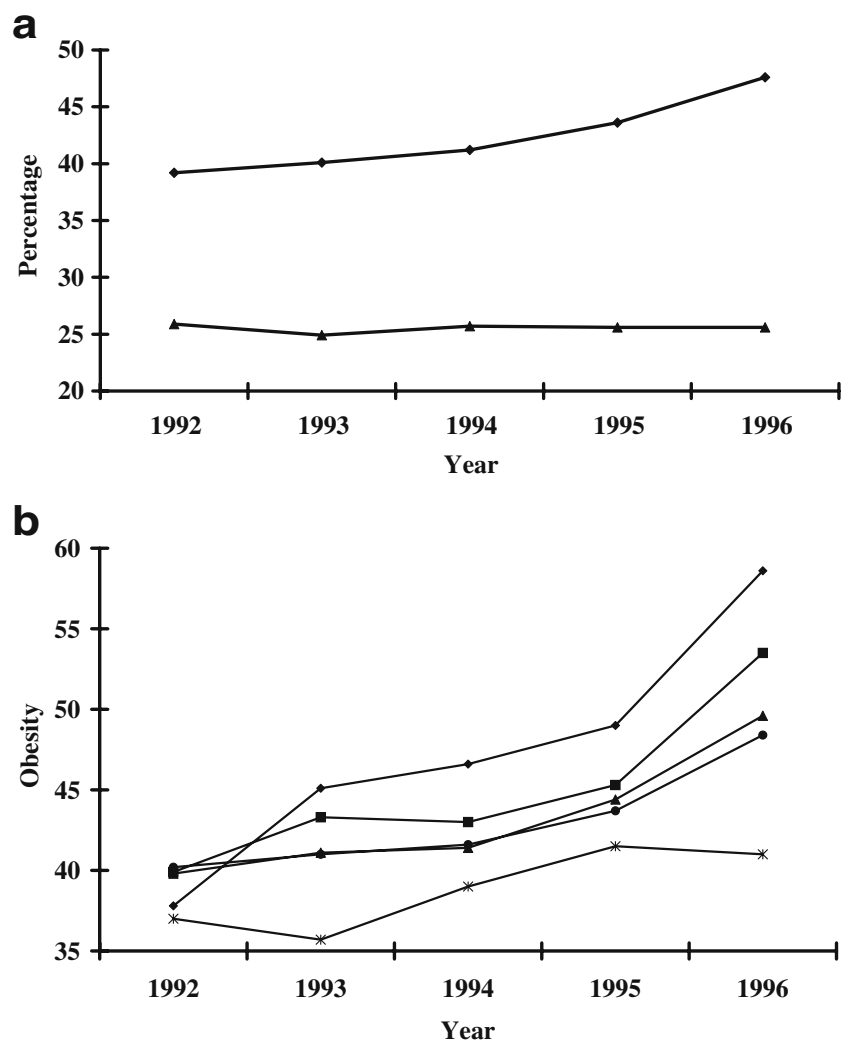

Fig. 1 a Trends in obesity (at interview; diamonds) and parental diabetes (triangles) in the incident cases of diagnosed type 2 diabetes in all patients. $p<0.001$ for obesity and $p>0.1$ for parental diabetes in all patients. $\mathbf{b}$ Trends in obesity according to age of onset of diabetes. Onset age is divided into $<35$ years (diamonds), 35-44 years (squares), 45-54 years (triangles), 55-64 years (spheres) and $\geq 65$ years $($ stars $)$ subgroup of onset age, sex and calendar year. The incidence trends were tested by linear regression, in which the incidence rate was the dependent variable and the calendar year was the independent variable. The incidence rate ratios between the year 1996 and the year 1992 were calculated using the incidence rates of 1992 as referent groups. A linear test for trend for the proportions of obesity and parental diabetes among the incident cases was performed for each group of onset age in the different sexes. Data were expressed as means (SD) or percentages, and $p<0.05$ was considered statistically significant.

\section{Results}

Table 1 shows the baseline characteristics of the incident cases during the years $1992-1996$ by sex. There were more women than men, and women were significantly older in onset age, had higher BMI at interview, but a lower percentage of parental diabetes. The proportion of obesity at interview was also higher in the women.

Table 2 shows the age- and sex-specific incidence rates and incident case numbers calculated for the years 19921996. Incidence increased with age, reaching a peak at the age of 55-64 years, and then decreased slightly. Except for the age groups of 45-54 and 55-64 in both sexes and the age groups of 35-44 and 55-64 in women, the trends of incidence rates increased significantly from 1992 to 1996. The respective incidence rate ratios between the years 1996 and 1992 are also shown in Table 2.

Figure 1 shows the trends of obesity at interview and parental diabetes in the incident cases. The trend of obesity at interview was highly significant for all age groups as a whole (Fig. 1a) and for different age groups (Fig. 1b) $(p<0.001)$. The trend of parental diabetes was not significant for the group as a whole (Fig. 1a, $p>0.1$ ), but showed different effects in different age groups $(p<0.05$ for the group $<35$ years with a decreasing trend; $p<0.05$ for the age groups $\geq 65$ and 55-64 years with increasing trends; and $p>0.1$ for the age groups $45-54$ and 35-44 years with nonsignificant trends).

\section{Discussion}

The findings demonstrated an increasing trend of diagnosed type 2 diabetes in Taiwan during 1992-1996. As a whole, a 1.8-fold increase in incidence was observed for both sexes (Table 2). The incidence rate ratios were higher in men than in women for all age groups (Table 2). Although the incidences were relatively low in the population aged $<35$ years, the rate ratios were highest in this age group (Table 2). A parallel increase in obesity at interview was observed in all age groups (Fig. 1), accompanying the trend 
of increasing incidence (Table 2). This was also most marked in the young population.

In Taiwan, obesity is becoming more common, especially in the young generation and the male gender [14-16]. In three surveys among schoolchildren aged 12-15 years in 1980-1982, 1986-1988 and 1994-1996, the respective prevalences of obesity were $12.4,14.8$ and $16.4 \%$ for boys and $10.1,11.1$ and $11.1 \%$ for girls [14]. Comparing the data obtained in the second [15] and third [16] National Nutrition and Health Survey in Taiwan, in 1986-1988 and 1993-1996, respectively, mean body weight increased by $1.2-7.0 \mathrm{~kg}$ in males and $0.9-9.0 \mathrm{~kg}$ in females, and BMI increased in all ages from 4 to $\geq 65$ years in both sexes. It is also interesting that obesity was more prevalent in men at younger ages, but more prevalent in women at older ages [16]. This partly explained the incidence rates being higher in males at younger ages and higher in females at older ages (Table 2).

Similarly, the incidence of diabetes increased in Pima Indians (by 50\% during the two 10-year periods 1965-1975 and 1975-1985) [17] and in other ethnic groups in the San Antonio Heart Study (the rate tripled over a 9-year period) in the USA [18]. The greatest increase was observed in adolescents aged 10-19 years in Cincinnati, Ohio (ten-fold, from 0.7 per 100,000 in 1982 to 7.2 per 100,000 in 1994) [19]. Although it is difficult to compare trends among different studies, the results of the present study suggest that the increase in incidence trends within a 5-year period in the Taiwanese population is probably among the highest rising trends.

Not all studies have reached similar conclusions. For example, in studies using registered prescriptions in Fyn County, Denmark, the increase in the number of diabetic patients was mainly due to the slightly decreasing mortality rate and the stable incidence $[4,5]$. However, the potential bias in this study included possible changes in detection rates and in prescription practices over the study period [2]. Furthermore, the declining mortality in Denmark would explain only a small proportion of the increasing prevalence of diabetes [3].

The major strength of the present study is the large sample size, and one implication is that the incidence trend of diagnosed diabetes in Taiwan could be well monitored by using the National Health Insurance database periodically, without having to follow a cohort for a long period of time. However, some limitations might exist; these are discussed below.

The results may have been limited by the exclusion of patients who did not join the insurance programme; alternatively, the patients may not have been labelled as having diabetes in the claim data submitted from the clinical setting to the Bureau of NHI. However, this is unlikely as the NHI programme is a country-wide health care system in Taiwan and has a high coverage rate. NHI is highly utilised by diabetic patients [11], and hospitals would report all cases of diabetes in the claim data if the patients had diabetes, in order to receive reimbursement. Also, it should be noted that the Bureau of NHI deters false reports of diagnosis by imposing severe penalties.

The incidence rates might have been underestimated because of the exclusion of undiagnosed diabetic patients. However, underestimation may have progressively become less likely over the 5 years as a result of the increasing focus on type 2 diabetes. Furthermore, the motivation of the diabetic patients for follow-up might have decreased with increasing diabetes duration. Thus, the estimated trend might have been somewhat overestimated. To minimise the impact of changes in diagnostic criteria for diabetes introduced by the American Diabetes Association in 1997 [10], which might falsely indicate an increase in diabetes, we limited our analyses to a period before 1997. Furthermore, the NHI programme cannot have had more than a marginal influence on the implementation of the diagnostic criteria as the programme was introduced at the end of the study period. This would also minimise the potential influence of policy changes on the observed results.

One might be concerned that the introduction of NHI in 1995 might have had an influence on the incidence of diagnosed diabetes. When we scrutinised the figures in Table 2, we found that the increasing trends actually occurred before the introduction of NHI in 1995, suggesting a minimal impact of the introduction of NHI in this study. However, such an influence might not happen suddenly and would not be obvious over several years after the introduction of NHI. Whether this important reshaping of the national health care system could have an effect on the number of cases of type 2 diabetes detected awaits further investigation.

The prevalence of diabetes could be different between different parts of Taiwan. Therefore, the use of a fixed correction factor to correct for the fractions of sampled populations might not be applicable if we aimed at the incidence of diabetes in a specific region of Taiwan.

Recall bias might be a problem given that the mean time periods between the diagnosis of diabetes and interview were $6.3,5.4,5.0,3.4$ and 2.5 years for patients diagnosed in the years 1992, 1993, 1994, 1995 and 1996, respectively $(p<0.05)$. However, when we analysed the time period from sampling to being interviewed, the percentages for $<1,1-$ $1.9,2-2.9,3-3.9$ and $\geq 4$ years were 19.7, 21.8, 26.4, 27.4 and $4.8 \%$, respectively. When we analysed the time period between diabetes diagnosis and the time of being interviewed, the percentages for the periods $<3,3-3.9,4-4.9,5-$ 5.9 and $\geq 6$ years were $34.0,19.7,21.3,15.4$ and $9.6 \%$, respectively. Therefore, only a small proportion of the 
patients were interviewed after a prolonged period of sampling or diabetes diagnosis. Furthermore, the year in which the NHI programme was introduced (1995) was an important landmark. We referred this landmark to the patients, and they actually had to recall only up to 3 years before or 1 year after the introduction of NHI. We therefore believe that recall bias had a limited impact on the results.

It was not possible to completely exclude cases of type 1 diabetes without confirmation by serological tests for antibodies or a pancreatic function test. Furthermore, some cases of secondary diabetes could not be ruled out. However, because the other classes of diabetes are rare, we believe that the results of this study mainly reflected the trends of diagnosed type 2 diabetes.

Although we used the BMI at interview, we believe that this would considerably overestimate the prevalence of obesity at diabetes diagnosis in those diagnosed in earlier years compared with those diagnosed in more recent years, because treatment with oral agents or insulin during the first 3-4 years was always accompanied by an increase in body weight [20]. On the other hand, the use of BMI at interview could have the advantage of minimising the chance of recall bias.

In summary, this study supports an increasing trend in the incidence of diagnosed type 2 diabetes in parallel with an increase in obesity at interview in patients in Taiwan. Taking into account the great morbidity and mortality associated with diabetes, there is an urgent need to take early action to halt this trend of an alarming increase, especially in the young generation, in whom obesity is a potential target of intervention.

Acknowledgements This study was supported by the Department of Health (DOH89-TD-1035) and the National Science Council (NSC90-2320-B-002-197, NSC-92-2320-B-002-156, NSC-93-2320-B-002071 and NSC-94-2314-B-002-142) of Taiwan. The authors thank the Department of Medical Research at the National Taiwan University Hospital for providing facilities, space and support for related studies.

\section{References}

1. Tai TY, Yang CL, Chang CJ et al (1987) Epidemiology of diabetes mellitus among adults in Taiwan, R.O.C. J Med Assoc Thai 70 (Suppl 2):42-48

2. Colagiuri S, Borch-Johnsen K, Glumer C, Vistisen D (2005) There really is an epidemic of type 2 diabetes. Diabetologia 48:1459-1463
3. Wareham NJ, Forouhi NG (2005) Is there really an epidemic of diabetes? Diabetologia 48:1454-1455

4. Green A, Stovring H, Andersen M, Beck-Nielsen H (2005) The epidemic of type 2 diabetes is a statistical artefact. Diabetologia 48:1456-1458

5. Stovring H, Andersen M, Beck-Nielsen H, Green A, Vach W (2003) Rising prevalence of diabetes: evidence from a Danish pharmaco-epidemiological database. Lancet 362:537-538

6. Tseng CH (2004) Mortality and causes of death in a national sample of diabetic patients in Taiwan. Diabetes Care 27:1605-1609

7. Tseng CH, Chong CK, Sheu JJ, Wu TH, Tseng CP (2005) Prevalence and risk factors for stroke in type 2 diabetic patients in Taiwan: a cross-sectional survey of a national sample by telephone interview. Diabet Med 22:477-482

8. Tseng CH, Chong CK, Heng LT, Tseng CP, Tai TY (2000) The incidence of type 2 diabetes mellitus in Taiwan. Diabetes Res Clin Pract 50(Suppl 2):S61-S64

9. Bonora E, Kiechl S, Willeit J et al (2004) Population-based incidence rates and risk factors for type 2 diabetes in white individuals: the Bruneck study. Diabetes 53:1782-1789

10. The Expert Committee on the Diagnosis and Classification of Diabetes Mellitus (1997) Report of the expert committee on the diagnosis and classification of diabetes mellitus. Diabetes Care 20:1183-1197

11. Lin T, Chou P, Lai MS, Tsai ST, Tai TY (2001) Direct cost of illness of patients with diabetes mellitus in Taiwan. Diabetes Res Clin Pract 54(Suppl 1):S43-S46

12. World Health Organization. The Asia-Pacific perspective: redefining obesity and its treatment. Available at: http://www.iotf.org (accessed 30 September 2005)

13. Department of Health, Taiwan Health and National Health Insurance Annual Statistics Information Service. Available at: http://www.doh.gov.tw/statistic/index.htm (accessed 30 September 2005)

14. Chu NF (2005) Prevalence of obesity in Taiwan. Obes Rev 6: 271-274

15. Kao MD, Huang MS, Tzeng NY, Lee NY, Shieh MJ (1991) The nutritional status in Taiwan: anthropometric measurement, 19861988. I. Body weight and body height [in Chinese]. J Chin Nutr Soc 16:63-84

16. Department of Health, Taiwan (1998) National nutrition and health survey in Taiwan, 1993-1996 [in Chinese]

17. Knowler WC, Pettitt DJ, Saad MF, Bennett PH (1990) Diabetes mellitus in the Pima Indians: incidence, risk factors and pathogenesis. Diabetes Metab Rev 6:1-27

18. Burke JP, Williams K, Gaskill SP, Hazuda HP, Haffner SM, Stern MP (1999) Rapid rise in the incidence of type 2 diabetes from 1987 to 1996: results from the San Antonio Heart Study. Arch Intern Med 159:1450-1456

19. Pinhas-Hamiel O, Dolan LM, Daniels SR, Standiford D, Khoury PR, Zeitler P (1996) Increased incidence of non-insulindependent diabetes mellitus among adolescents. J Pediatr 128:608-615

20. Turner RC, Cull C, Holman R (1996) United Kingdom Prospective Diabetes Study 17: a 9-year update of a randomized, controlled trial on the effect of improved metabolic control on complications in non-insulin-dependent diabetes mellitus. Ann Intern Med 124:136-145 